\title{
Clinical management and therapy of idiopathic recurrent pericarditis
}

\begin{abstract}
Recurrent pericarditis is defined when pericarditic chest pain reappears after a symptom-free period of at least 4 to 6 weeks and after completion of full-dose anti-inflammatory therapy. Idiopathic pericarditis is the commonest etiology. The diagnosis of idiopathic cases is essentially an exclusion diagnosis, supported by a typical clinical course. The diagnosis is based on the association of typical symptoms and signs: mainly pericarditic chest pain plus pericardial rubs or electrocardiographic alterations or pericardial effusion. The optimal regimen for recurrences includes combination of non-steroidal anti-inflammatory agents, colchicine, and corticosteroids. In the resistant forms, immunotherapy (azathioprine, intravenous immunoglobulins, and particularly anakinra) has shown to be effective. The long term outcome of idiopathic recurrent pericarditis is good, with no evolution towards constrictive form.
\end{abstract}

Keywords: Recurrence; Pericarditis; Anti-Inflammatory Agents, Non-Steroidal;Immunotherapy; Interleukin 1 Receptor Antagonist Protein

Gestione clinica e terapia della pericardite ricorrente idiopatica

CMI 2018; 12(1): 1-9

https://doi.org/10.7175/cmi.v12i1.1339

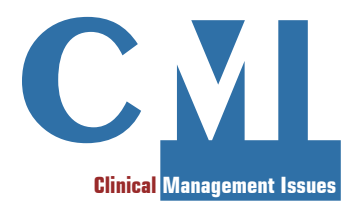

Clinical Management
Internal Medicine,

Hospital Papa

Giovanni XXIII,

Bergamo, Italy

\section{INTRODUCTION}

Acute pericarditis is an inflammatory pericardial syndrome, defined when at least two of the four following criteria are satisfied:

- Chest pain, typically retrosternal, worsened in supine position and with deep inspiration, relieved in the upright position;

- Pericardial friction rub (unfortunately this sign is present only in one third of all patients);

- Typical electrocardiographic features, namely PR depression and diffuse concave ST segment elevation;

- New onset or worsening pericardial effusion.

Other supportive findings for diagnosis are fever, elevation of markers of inflammation $(\mathrm{C}$-reactive protein-CRP, erythrocyte sedimentation rate-ESR, white blood cell count) or evidence of pericardial inflammation detected by imaging technique, for $\mathrm{ex}^{-}$ ample cardiac magnetic resonance (CMR).[1]

Recurrent pericarditis is defined as a relapse of disease after a documented first episode followed by a symptom-free interval of 4-6 weeks or longer, arbitrarily defined by experts and corresponding to the completion and usual duration of anti-inflammatory therapy. Diagnosis of recurrence is established according to the same criteria as those used for acute pericarditis.[1]

The recurrence rate after an initial episode of pericarditis ranges from 15 to $30 \%$ and may increase to $50 \%$ after a first recurrence in patients not adequately treated.[1] (ClinicalTrials.gov registration number: NCT00128414 [2]; ClinicalTrials.gov registration number: NCT00235079 [3]).
Corresponding author Andrea Assolari andrea.assolari@tiscali.it

Received: 15 November 2017 Accepted: 6 December 2017 Published: 19 January 2018 
Table I. Recommended investigations for pericarditis. [1]

$\mathrm{ACE}=$ angiotensinconverting enzyme; ANA $=$ antinuclear antibodies; $\mathrm{ANCA}=$ antineutrophil cytoplasm antibodies; $\mathrm{CRP}=\mathrm{C}$-reactive protein; ENA $=$ antiextractable nuclear antigens; $\mathrm{ESR}=$ erythrocyte sedimentation rate FMF $=$ familial Mediterranean fever; $\mathrm{RF}=$ rheumatoid factor; TRAPS = tumor necrosis factor receptor associated periodic syndrome

\begin{tabular}{|c|c|c|}
\hline \multicolumn{2}{|c|}{ Level } & Investigation \\
\hline \multicolumn{2}{|l|}{ First level (all cases) } & $\begin{array}{l}\text { - Markers of inflammation (CRP, ESR, white blood cell count) } \\
\text { - Renal function and liver tests, thyroid function } \\
\text { - Markers of myocardial lesion (troponin) } \\
\text { - ECG } \\
\text { - Echocardiography } \\
\text { - Chest X-ray }\end{array}$ \\
\hline \multirow{5}{*}{$\begin{array}{l}\text { Additional testing } \\
\text { directed to } \\
\text { specific etiologies } \\
\text { according to clinical } \\
\text { presentation }\end{array}$} & $\begin{array}{l}\text { Probable viral } \\
\text { infections }\end{array}$ & $\begin{array}{l}\text { - Viral serological tests are not recommended, with the } \\
\text { possible exception of anti-HIV, anti-HCV, HBsAg }\end{array}$ \\
\hline & $\begin{array}{l}\text { Probable } \\
\text { tuberculosis }\end{array}$ & $\begin{array}{l}\text { - Quantiferon, Mantoux } \\
\text { - Culture and PCR in sputum or other biological fluids } \\
\text { - Chest CT Scan } \\
\text { - Acid-fast bacilli staining, Mycobacterium cultures, PCR for } \\
\text { genome on pericardial fluid }\end{array}$ \\
\hline & $\begin{array}{l}\text { Probable } \\
\text { neoplasm }\end{array}$ & $\begin{array}{l}\text { - Specific neoplasm markers } \\
\text { - Chest and abdomen CT scan, PET } \\
\text { - Cytology of pericardial fluid or pericardial biopsy }\end{array}$ \\
\hline & $\begin{array}{l}\text { Probable } \\
\text { autoimmune } \\
\text { disease }\end{array}$ & - ANA, ENA, ANCA, ACE, RF, ferritin \\
\hline & $\begin{array}{l}\text { Probable } \\
\text { autoinflammatory } \\
\text { conditions } \\
\text { (periodic fevers) }\end{array}$ & - FMF and TRAPS mutations \\
\hline \multicolumn{2}{|c|}{$\begin{array}{l}\text { Second level (first level not sufficient for } \\
\text { diagnostic purpose) }\end{array}$} & $\begin{array}{l}\text { - Computed tomography and/or cardiac magnetic resonance } \\
\text { - Pericardiocentesis (analysis of pericardial fluid) }\end{array}$ \\
\hline
\end{tabular}

Patients at risk for recurrent attacks are patients with incomplete response to non-steroidal anti-inflammatory drugs (NSAIDs), patients with high fever or large effusion at presentation or elevated $\mathrm{C}$ reactive protein despite at least one week of treatment.[1] Other treatment-related risk factors are: inadequate initial full dose of NSAIDs regimen or too rapid drug tapering, corticosteroids use, lack of colchicine, discontinuation of two classes of drugs together.

Other known mechanisms classically involved include exacerbations of the initial infections or reinfections and unrecognized cause untreated. Some factors that may trigger the recurrence are acute respiratory infections, physical efforts, cold temperature exposition.[4]

\section{CLINICAL MANAGEMENT}

The main symptom of recurrent pericarditis is chest pain, similar in characteristics to the pain suffered in the first episode, but in most cases less powerful, more attenuated. The chest pain of pericarditis is typically retrosternal in location, sudden in onset, pleuritic in nature. Chest pain is worse when the patient is supine and improves when he or she sits upright. Chest pain is exacerbated by inspiration. A prodromal of fever, malaise, and myalgia can be common.[5]

Auscultation, electrocardiograph (ECG), echocardiography, chest $\mathrm{X}$-ray, and routine blood tests are first-level investigations, recommended in all cases (Table I).[1]

During physical examination some patients have an audible friction rub.

ECG can show upward concave ST-segment elevation and PR-segment depression. In recurrent pericarditis, nonspecific alterations of ventricular repolarization or abnormalities of T-waves are common.

Transthoracic echocardiography can reveal the presence of a new onset or worsened pericardial effusion.

Chest X-ray provides information with regard to cardiac size and the presence of pulmonary pathology, pleural effusion and hilar and mediastinal enlargement.

Blood tests to perform are white cell count, ESR and CRP, renal function and liver tests, thyroid function, markers of myocardial injury.[1] Unfortunately, no specific diagnostic marker is available for pericarditis. However, most patients with pericarditis show high levels of markers of inflammation. 
$\mathrm{CRP}$ and ESR and the other parameters of inflammation, even though not specific, are very important to define the intensity of the inflammatory process and select appropriate length of therapy. For example, CRP dosage can be useful to confirm the diagnosis and differentiate true relapses from atypical chest pains. Persistent elevation of CRP may indicate ongoing inflammation and requires prolonged therapies. Monitoring of CRP may be useful to follow the disease activity and to guide the appropriate length of therapy, with continuation of the full dose until CRP normalization, at which time tapering may be considered. During the therapy, in addition to the monitoring of side effects, a monthly check of CRP, blood cell count, renal and hepatic function, inflammatory index is strongly recommended.[6]

The diagnostic work-up of recurrent pericarditis may provide the use of second-level investigations in order to identify specific etiologies or detect if significant pericardial inflammation persists despite therapy. The identification of an underlying etiology is particularly important because it allows to start a targeted therapy.[1,7,8]

Many diseases can cause pericarditis, but, for a practical approach, causes can be divided in two major categories: infectious (any kind of microorganisms) and non-infectious causes (autoimmune, neoplastic, metabolic, traumatic or iatrogenic, drug-related, and miscellaneous).[1]

In developed countries and in immunocompetent patients, recurrent pericarditis is idiopathic or viral in most cases (80-85\%). Viral infections causing pericarditis include coxsackievirus, echovirus, adenovirus, parvovirus, human herpes virus 6 , but specific viral serological tests are considered futile. The diagnosis of idiopathic cases is essentially an exclusion diagnosis, supported by a typical clinical course. $[1,7,8]$

In patients with repeated relapses and in presence of certain clinical features, it is important to exclude some etiologies that may not have been identified in previous assessments: tuberculosis, systemic inflammatory diseases, such as a connective tissue disease or neoplastic diseases.[1]

In this regard, specific blood tests, computed tomography $(\mathrm{CT})$, positron emission tomography (PET), and cardiac magnetic resonance (CMR) may be useful.

$\mathrm{CT}$ is considered a complementary imaging modality. $\mathrm{CT}$ generally provides anatomic information and defines the extent of pericardial calcification, but with administration of iodinated contrast medium it is possible to detect pericardial inflammation because of the enhancement of the inflamed pericardium after contrast injection.[9] CT, if extended to thorax and abdomen, may detect neoplastic masses or lymphadenopathies, thus suggesting possible tuberculosis.

In selected cases, PET, preferably in combination with $\mathrm{CT}$, can be indicated to depict the metabolic activity of pericardial disease. Pericardial uptake of tracer in patients with solid cancers and lymphoma is indicative of malignant pericardial involvement, thus providing key information on the diagnosis, staging, and assessment of the therapeutic response. PET/CT is also of value in identifying the nature of inflammatory pericarditis. For example, tuberculosis pericarditis yields higher tracer uptakes than the idiopathic forms.[10]

Cardiac magnetic resonance is a very helpful second-level imaging technique to study pericardial tissue. CMR assesses pericardial thickness and can have a significant role in the evaluation of pericardial inflammation. Following intravenous administration of gadolinium, late gadolinium enhancement can show the extension of the inflammation into the surrounding epicardial fat, suggesting a severe inflammation. Some patient, especially those with complicated or recurrent pericarditis, may benefit from this exam primarily directed at identifying if the patient still has significant pericardial inflammation. CMR is indicated also in patients in whom the presence of active pericardial inflammation is uncertain: delayed pericardial enhancement at CMR may favor continued or intensified anti-inflammatory treatment. On the other hand, if CMR does not show delayed pericardial enhancement, then tapering of medications may continue, and other diagnosis may be considered. CMR is useful also to show myocardial inflammatory involvement, fibrosis, and constrictive evolution. Unfortunately, the technique has some important disadvantages: limited availability, high costs, and also the need for breath-holding and regular heart rhythms to get a better quality of the picture.[4]

As stated in the most updated European Society of Cardiology (ESC) guidelines,[1] pericardiocentesis should be used just in case of cardiac tamponade and suspicion of neoplastic or bacterial etiology as absolute indications. Moreover, it should be considered in case of chronic (> 3 months) large pericardial effusions. 


\section{THERAPY}

The treatment of patients with recurrences is not very different to the treatment of a first episode of acute pericarditis. Aspirin or NSAIDs remain the mainstay of therapy. Colchicine is recommended on top of standard anti-inflammatory therapy in order to improve remission rates and prevent recurrences. In case of incomplete response to NSAIDs and colchicine, corticosteroids may be used, but they should be added at low-to-moderate doses.[1]

\section{Aspirin and NSAIDs}

Aspirin and NSAIDs remain the mainstay of treatment.[1,7,8] The specific drug selected is not important: the choice should be based on the physician's experience as well as on the history of efficacy and tolerability in the single patient. An NSAID that was effective in a previous attack should be the favorite choice. Ibuprofen and aspirin are the most used. Indomethacin is perhaps the most powerful anti-inflammatory drug. Also comorbidities are important: for example, aspirin is the favored choice in patients with ischemic heart disease or when a patient is already on aspirin or needs anti-platelet treatment. Also naproxen is an alternative in these situations. Indomethacin and other NSAIDs should be avoided in patients with coronary artery disease.

During an acute attack, a practical tip in a hospitalized patient is the administration of aspirin or NSAIDs intravenously, above all if the patient has intensive pain, high fever with associated nausea or vomiting. For example, in our clinical experience we adminis- ter indomethacin $100 \mathrm{mg}$ in $250 \mathrm{ml}$ of saline in continuous infusion in a day. Therefore, after 3-5 days of intravenous indomethacin, if the patient is better, i.v. indomethacin can be switched to oral therapy. Ketorolac is another anti-inflammatory widely used intravenously in the hospital setting, but it can only be used for a short time and, on the contrary, patients with relapsing pericarditis need the therapy for a long time.

Particular attention should be paid in using the highest tolerable dose of each medication and reassuring a continuous antiinflammatory coverage throughout the day. A common mistake is to use too low doses. Aspirin should be used at the dose of 1.5 to $4 \mathrm{~g} /$ day; ibuprofen $1800-2400 \mathrm{mg} / \mathrm{day}$; indomethacin 75-150 mg/day (Table II).

The administration of NSAIDs should be well distributed during the day. For example, with regard to aspirin, ibuprofen, or indomethacin, each dose should be taken precisely every 8 hours in order to guarantee a full 24-hour coverage. [8,11]

The duration of optimal treatment and the need to reduce the dose have not been tested in clinical trials. It is well established that the full dose regimen should be offered at least until normalization of CRP values and initial clinical remission. This may take months, especially in patients with history of recurrent pericarditis. Generally, respect the first episode of acute pericarditis, the length of treatment should be more extended.

NSAIDs' side effects are well known. The most serious are ulcers, bleeding, and kidney failure.

Aspirin use should be more cautious in patients with initial impaired renal function,
Table II. Commonly prescribed anti-inflammatory therapies and dosages for recurrent pericarditis. [1] *Valid for all alternatives

\begin{tabular}{|c|c|c|c|}
\hline Drug & Attack dose & Length of treatment: & Tapering \\
\hline Aspirin & $\begin{array}{l}500-1500 \mathrm{mg} \text { every } 8 \text { hours } \\
(1.5-4 \mathrm{~g} / \mathrm{d})\end{array}$ & $\begin{array}{l}\text { First attack: 2-4 weeks } \\
\text { Recurrences: several } \\
\text { weeks/months. }\end{array}$ & $\begin{array}{l}\text { Decrease the total daily } \\
\text { dose by } 250-500 \mathrm{mg} \\
\text { every 2-4 weeks }\end{array}$ \\
\hline Ibuprofen & $\begin{array}{l}600-800 \mathrm{mg} \text { every } 8 \text { hours } \\
\text { (1800-2400 mg daily) }\end{array}$ & $\begin{array}{l}\text { The optimal length } \\
\text { of treatment is } \\
\text { debatable, and CRP }\end{array}$ & $\begin{array}{l}\text { Decrease the total daily } \\
\text { dose by } 200-400 \mathrm{mg} \\
\text { every 2- } 4 \text { weeks }\end{array}$ \\
\hline Indomethacin & $\begin{array}{l}25-50 \text { mg every } 8 \text { hours } \\
\text { (75-150 mg daily) }\end{array}$ & $\begin{array}{l}\text { a marker of disease } \\
\text { activity to guide } \\
\text { management and length }\end{array}$ & $\begin{array}{l}\text { Decrease the total daily } \\
\text { dose by } 25 \mathrm{mg} \text { every } \\
2-4 \text { weeks }\end{array}$ \\
\hline Naproxen & $\begin{array}{l}250-500 \mathrm{mg} \text { every } 12 \text { hours; maximal } \\
\text { daily dose } 1500 \mathrm{mg} \text { for limited } \\
\text { time period ( }<6 \text { months). Dosage } \\
\text { expressed as naproxen base; } 200 \mathrm{mg} \\
\text { naproxen base is equivalent to } \\
220 \mathrm{mg} \text { naproxen sodium }\end{array}$ & $\begin{array}{l}\text { of treatment. Gradual } \\
\text { tapering (every 1-2 } \\
\text { weeks and only if the } \\
\text { patient is asymptomatic } \\
\text { and CRP is normal) is } \\
\text { recommended }\end{array}$ & $\begin{array}{l}\text { Decrease the total daily } \\
\text { dose by } 125-250 \mathrm{mg} \\
\text { every } 1-2 \text { week }\end{array}$ \\
\hline
\end{tabular}


erosive gastritis, peptic ulcer, gout, platelet, and bleeding disorders. NSAIDs use should be more cautious in patients with cardiac dysfunction, hypertension, renal or hepatic impairment, epilepsy, and patients receiving anticoagulants. However, in most patients these medications are taken for months without causing significant side effects. A proton pump inhibitor should be prescribed to all patients under aspirin or NSAIDs treatment. $[8,11]$

\section{Colchicine}

In case of pericarditis, colchicine is always indicated, even in the first attack, but especially in case of recurrence. Colchicine should be added to the NSAIDs, and should not replace them.[1]

Colchicine therapy is able to improve the response to traditional anti-inflammatory therapy and reduce the relapse rate of at least $50 \% .[2,3]$

Side effects are gastrointestinal (up to $10 \%$ of cases), including nausea, vomiting, diarrhea, and abdominal pain, usually being a common cause of drug withdrawal; generally mild, they may resolve with dose reduction. Weight-adjusted doses may reduce these side effects. Less common side effects include elevation of transaminases. Other anecdotal side effects are bone marrow suppression (less than 1\%) and reversible alopecia $(0.6 \%)$. Colchicine interacts with macrolide antibiotics, in particular clarithromycin. A practical advice is to halve the dose of colchicine while taking clarithromycin.

During the therapy with colchicine, it is recommended to monitor blood cell count, renal function, and transaminases at least at baseline and after a month of therapy. These exams are routinely performed in patients with many recurrences.

In order to improve patients' compliance and minimize the risk of side effects, colchicine should be administered in low, weight-adjusted doses $(0.5 \mathrm{mg}$ once daily for patients $<70 \mathrm{~kg}, 1 \mathrm{mg}$ once daily or $0.5 \mathrm{mg}$ twice daily for patients $>70 \mathrm{~kg}$ ). A loading dose is not necessary. Dose adjustment is mandatory for patients with renal impairment, debilitated patients, and elderly patients.[1-3]

The duration of therapy is at least 6 months, but, if recurrences are frequent and colchicine is well tolerated, the duration can reach some years. At this point, discontinuation is discussed with the patient, explaining that cases of recurrence after colchicine discontinuation have been reported. Even for colchicine, suspension should not be abrupt, but gradual.[2,3,12]

\section{Corticosteroids}

Corticosteroids are not recommended as first-line approach. It was shown that when they were used as first and unique therapy, though they produced a fast and satisfactory clinical response, they created a kind of addiction, a chronicity, and the patient became dependent.

Corticosteroids should be considered only in specific situations and the indications for their use are limited, for example in case of incomplete response to aspirin/NSAIDs and colchicine. In this case, they should be added at low-moderate doses as triple therapy in order to achieve better control of symptoms. NSAIDs are really contraindicated in case of true allergy or intolerance, renal failure and advanced kidney disease, pregnancy beyond the $20^{\text {th }}$ week of gestation, recent peptic ulcer or gastrointestinal bleeding, oral anticoagulant therapy when the bleeding risk is considered high or unacceptable. Corticosteroids are also correctly used when there is an autoimmune disease. Indeed, low doses of steroids are commonly used to treat serositis.[1] Even in these cases, colchicine should be associated.

Although guidelines and reviews suggest limiting the use of these drugs, they are commonly prescribed, especially during recurrences.

Low-to-moderate doses $(0.2-0.5 \mathrm{mg} / \mathrm{kg} /$ day of prednisone or dose equivalent, in most patients generally 5-10 mg/day) are sufficient in most patients and the dose should almost never exceed $25 \mathrm{mg} /$ day.[13]

Side effects are numerous and well known: weight gain, hypertension, diabetes, immunosuppression with masked or latent opportunistic infections, osteoporosis, acute myopathy, elevated intraocular pressure, cataract, central nervous system effects ranging from euphoria to psychosis. In order to prevent osteoporosis, supplementation of calcium and vitamin $\mathrm{D}$ and bisphosphonates, when indicated, is performed. With the aim of preventing weight increase, hyperglycemia, and liquid retention, some rules must be respected, and in particular diet restriction is necessary.

After having obtained a clinical response, corticosteroids tapering should be very 


\begin{tabular}{|l|l|}
\hline \multicolumn{1}{|c|}{ Starting dose: 0.2-0.5 mg/kg/day } & \multicolumn{1}{|c|}{ Tapering } \\
\hline$>50 \mathrm{mg}$ & $10 \mathrm{mg} /$ day every $1-2$ weeks \\
$25-50 \mathrm{mg}$ & $5-10 \mathrm{mg} /$ day every $1-2$ weeks \\
$15-25 \mathrm{mg}$ & $2.5 \mathrm{mg} /$ day every 2-4 weeks \\
$<15 \mathrm{mg}$ & $1.25-2.5 \mathrm{mg} /$ day every 2-6 weeks \\
\hline
\end{tabular}

Table III. Tapering of corticosteroids (dosage information for prednisone).[1]

gradual, over months as in rheumatic polymialgia. Table III shows a schedule about steroid reduction based on the dose used.[1]
A critical threshold is a $10-15 \mathrm{mg} /$ day dose of prednisone or equivalent. At this threshold, decrements should be very slow, for example 1-2.5 $\mathrm{mg}$ at intervals of 2-6 weeks. Each tapering should be performed only with normal CRP and in the absence of symptoms. In case of recurrence during tapering, an important advice is not to increase the dose of steroid yet, but eventually split in 2 half doses controlling the symptoms, adding NSAIDs every 8 hours full dosage or intravenously, adding analgesics and reassuring the patient about duration of clinical disorders, generally only few days.[1]

\section{Triple therapy: a wand}

A protocol including non-steroidal antiinflammatory drugs at high dosage, colchicine, corticosteroids at low doses, reassurance, and close clinical monitoring generally allow patients to obtain a good control of the disease. [1,14]

After obtaining a complete response, tapering should be done with a single class of drugs at a time. We suggest this order of discontinuation: steroids first, NSAIDs second, and at last colchicine. Such process should be strictly individualized according to individual intolerability and renal function, as in elderly people. Since steroid discontinuation may require from 2 to 10 months, during this time, according the clinical condition, NSAIDs can be used at low or intermediate doses and only temporarily increased at high doses. Since colchicine is generally well tolerated, it is the last drug to be stopped. In the acute attack, analgesics, like acetaminophen or tramadol, can be added to control the pain.[1]

\section{Immunotherapy}

In patients with recurrent pericarditis despite the therapy with NSAIDs, cortico- steroids, and colchicine, the most updated ESC guidelines [1] suggest to consider the use of azathioprine, high-dose intravenous immunoglobulins, and anakinra.

Azathioprine is indicated for patients who require unacceptably high long-term doses of corticosteroids.[1] In these conditions, azathioprine is used as a steroid-sparing agent in order to control the disease at longterm follow up. On the contrary, it is not useful in the resolution of an acute attack. Azathioprine is a purine analog that inhibits lymphocytes proliferation. It is commonly used in autoimmune diseases. Data concerning azathioprine as therapy of recurrent pericarditis are scarce and based solely on adults' case reports.[16] The treatment dose for recurrent pericarditis is $2 \mathrm{mg} / \mathrm{kg}$ daily. The major side effect is myelosuppression, which resolves by reducing the dose in about one week. Other side effects are transient hepatotoxicity and gastrointestinal symptoms.

Intravenous immunoglobulins (IVIGs) are used for their immunomodulatory and immunosuppressive effects.[1] IVIGs have a role in the resolution of acute attacks and as steroid-sparing agent. The most commonly used dose ranges from 400 to $500 \mathrm{mg} / \mathrm{kg}$ per day for 5 days. Repeated cycles may be required, typically at monthly intervals. The main disadvantages of this treatment are costs and possible safety problems related to the use of plasma-derived substances. Fortunately, adverse reactions are very rare, mild, and transient. Care must be taken in the infusion rate.

Anakinra is a recombinant form of interleukin-1 (IL1) receptor antagonist and acts by antagonizing the biological effects of IL1, which is a pro-inflammatory cytokine. A recent study, AIRTRIP, has shown that anakinra has a spectacular effect in severe, cortico-dependent and colchicineresistant idiopathic recurrent pericarditis (ClinicalTrials.gov registration number: NCT02219828 [16]). It is important to select the right patients, that are those with very active and inflammatory disease, fever, strikingly elevated CRP, pleural effusions. Anakinra is not indicated in patients with ambiguous recurrences with normal CRP. The initial dose is $100 \mathrm{mg} / \mathrm{day}$; it is administered subcutaneously. The most common adverse effect is a local reaction at the injection site. For this reason, patients should be informed in advance. Local skin reactions disappear over one months or after topical steroids treatment and systemic antihista- 
mines. Warming the syringe to room temperature before use is advisable, along with application of a cold pack to the injection site approximately 2 to 3 minutes before and immediately after the injection to prevent local reactions. Other adverse effects are mild reversible elevation of transaminases and increased risk of infection. Neutropenia has been reported in $5 \%$ of cases, but without evidence of association with clinical events. The contraindications of its employment are acute infections, immunosuppression and neutropenia, hypersensitivity or a previous allergic reaction, pregnancy, and breastfeeding. The main advantages of anakinra are rapid onset of effect (few days) and the capability to allow quick withdrawal of corticosteroids and then NSAIDs (some weeks).

In our experience, the duration of therapy is at least three months at full dose. After obtaining remission with anakinra, a very gradual tapering is suggested (for example -100 $\mathrm{mg} /$ week every month till $300 \mathrm{mg} /$ weekly, and then $-100 \mathrm{mg} /$ week every 2-3 months), with concomitant colchicine therapy, avoiding corticosteroids. This indication is due to the fact that unfortunately recurrences are common also after anakinra discontinuation.

\section{Pericardiectomy}

If all drugs fail, the last option is the surgical removal of the pericardium in experienced surgical centers. The role of interventional and surgical techniques for the treatment of pericarditis is controversial, with the only exception of constrictive pericarditis. Fortunately, nowadays pericardiectomy is rarely required and should be regarded as the last resort in refractory pericarditis cases presenting with recurrent tamponade, and in patients unable to tolerate the aforementioned conventional treatment.[1] Table IV shows the therapeutic algorithm for recurrent pericarditis.

\section{CONCLUSION}

Recurrent pericarditis is often a frustrating disease, for both the patient and the physician. Indeed, recurrences are a common cause of concern for the fear of a possible evolution towards constriction or heart disease. In this regard, an analysis of cases of idiopathic recurrent pericarditis has shown no cases of constrictive pericarditis even after a long-term follow up [17]. A low risk $(0.4 \%)$ of constriction exists just after

\begin{tabular}{|l|l|}
\hline \multicolumn{1}{|c|}{ Line } \\
$\begin{array}{l}\text { First line } \\
\text { Second line }\end{array}$ & $\begin{array}{l}\text { Aspirin or NSAID + colchicine } \\
\text { Low-dose corticosteroids (in case of contraindication to } \\
\text { aspirin/NSAID/colchicines or in case of incomplete response } \\
\text { to aspirin/NSAID/colchicine) } \\
\text { Anakinra or azathioprine or IVIG }\end{array}$ \\
$\begin{array}{l}\text { Third line } \\
\text { Fourth line }\end{array}$ & Pericardiectomy \\
\hline
\end{tabular}

a first (and thus non-recurrent) episode of pericarditis.

Therefore, despite compromising the quality of life, idiopathic recurrent pericarditis has an overall good long-term prognosis without significant risk of constrictive pericarditis, heart disease evolution, or death $[17,18]$. Cardiac tamponade is rare and generally occurs at the beginning of the disease.

In conclusion, if patients with recurrent pericarditis are treated with a multi-drug protocol in which NSAIDs are used at recommended dosages, colchicine is added if tolerated, steroids are tapered very slowly with a close clinical monitoring, the clinical course and quality of life will show improvement. For those patients who do not respond to this protocol, anakinra can be a drug with spectacular results. An important component of therapy is reassurance and explanation of the nature of the disease and its likely course.

Key points in the management of recurrent pericarditis

- Recurrent pericarditis generally has good prognosis, implies a normal life, and presents no risk of evolution in constrictive pericarditis

- Aspirin and NSAIDs are the mainstay of treatment and are recommended at full doses, well distributed, intravenously if necessary, until complete symptoms resolution

- Colchicine at low dose (0.5-1 mg/day) is recommended as an adjunct to aspirin/NSAIDs. Colchicine therapy for long duration should be considered in some cases

- Corticosteroid therapy is not recommended as first-line approach. Low-dose corticosteroids should be considered in case of contraindication to aspirin/NSAID/colchicine or in case of incomplete response to aspirin/NSAID/colchicine

- $C R P$ dosage should be considered to guide the treatment duration and assess the response to therapy

- After CRP normalization and symptoms resolution, a gradual tapering of therapies should be considered, in this order: first corticosteroids, second NSAIDs or aspirin, third colchicine

- Drugs such anakinra, azathioprine, or IVIG may be considered in case of corticosteroid-dependent and colchicine-resistant recurrent pericarditis 


\section{Funding}

This article has been published without the support

of sponsors.

Conflicts of interests

$A B$ received unrestricted research grants by

Acarpia, Sobi, and Lilly. The other authors declare

they have no competing financial interests

concerning the topics of this article.

The commonest misunderstandings

- Low doses of NSAIDs, only orally or not well distributed to cover the day

- High dose of colchicine: Diarrhea and discontinuation

- Colchicine in monotherapy during the acute attack: It should be always added to an antiinflammatory drug

- Corticosteroids in monotherapy: Side effects and recurrences at discontinuation

- Rapid tapering of steroids: Tapering should be very gradual, over months, only done after symptoms resolution with normalization of CRP. If symptoms recur during therapy tapering, the management should consider not to increase the dose of corticosteroids, but control the symptoms increasing to the maximum the dose of aspirin or NSAIDs and adding analgesics.

- Automatic tapering not depending on CRP normalization.

\section{REFERENCES}

1. Adler Y, Charron P, Imazio M, et al. The task force for the diagnosis and management of pericardial diseases of the European society of cardiology (ESC) 2015 ESC Guidelines for the diagnosis and management of pericardial diseases. Eur Heart J 2015; 36: 2921-64; https:// doi.org/10.1093/eurheartj/ehv318

2. Imazio M, Brucato A, Cemin R, et al. Colchicine for recurrent pericarditis (CORP) a randomized trial. Ann Intern Med 2011; 155: 409-14; https://doi.org/10.7326/0003-4819155-7-201110040-00359

3. Imazio M, Belli R, Brucato A, et al. Efficacy and safety of colchicine for treatment of multiple recurrences of pericarditis (CORP-2): a multicenter, double-blind, placebo-controlled, randomized trial. Lancet 2014; 383:2232-7; https://doi.org/10.1016/S0140-6736(13)62709-9

4. Cremer PC, Kumar A, Kontzias A, et al. Complicated Pericarditis: understanding risk factors and pathophysiology to inform imaging and treatment. J Am Coll Cardiol 2016; 68: 2311-28; https://doi.org/10.1016/j.jacc.2016.07.785

5. Imazio M, Gribaudo E, Gaita F. Recurrent pericarditis. Prog Cardiovasc Dis 2017; 59: 360-8; https://doi.org/10.1016/j.pcad.2016.10.001

6. Imazio M, Brucato A, Maestroni S, et al. Prevalence of C-reactive protein elevation and time course of normalization in acute pericarditis: implications for the diagnosis, therapy, and prognosis of pericarditis. Circulation 2011; 123: 1092-7; https://doi.org/10.1161/ CIRCULATIONAHA.110.986372

7. Imazio M, Gaita F. Diagnosis and treatment of pericarditis. Heart 2015; 101: 1159-68; https:// doi.org/10.1136/heartjnl-2014-306362

8. Imazio M, Spodick DH, Brucato A, et al. Controversial issues in the management of pericardial diseases. Circulation 2010; 121: 916-28; https://doi.org/10.1161/ CIRCULATIONAHA.108.844753

9. Maggiolini S, De Carlini CC, Ferri LA, et al. The role of early contrast-enhanced chest computer tomography in the etiological diagnosis of patients presenting with cardiac tamponade or large pericardial effusion. Euro Heart J Cardiovasc Imaging 2016; 17: 421-8; https://doi.org/10.1093/ ehjci/jev225

10. Lobert P, Brown RK, Dvorak RA, et al. Spectrum of physiological and pathological cardiac and pericardial uptake of FDG in oncology PET/CT. Clin Radiol 2013; 68: 59-71; https:// doi.org/10.1016/j.crad.2012.09.007 
11. Imazio M, Brucato A, Trinchero R, et al. Individualized therapy for pericarditis. Expert Rev Cardiovasc Ther 2009; 7: 965-75; https://doi.org/10.1586/erc.09.82

12. Imazio M, Brucato A, Cemin R, et al. A randomized trial of colchicines for acute pericarditis. N Engl J Med 2013; 369: 1522-8; https://doi.org/10.1056/NEJMoa1208536

13. Imazio $M$, Brucato $A$, Cumetti $D$, et al. Corticosteroids for recurrent pericarditis. High versus low doses: a nonrandomized observation. Circulation 2008; 118: 667-71; https://doi.org/10.1161/ CIRCULATIONAHA.107.761064

14. Brucato A, Brambilla G, AdlerY, et al. Therapy for recurrent acute pericarditis: a rheumatological solution? Clin Exp Rheumatol 2006; 24: 245-50

15. Vianello F, Cinetto F, Cavraro M, et al. Azathioprine in isolated recurrent pericarditis; a single centre experience.Int J Cardiol 2011; 147: 477-8; https://doi.org/10.1016/j.ijcard.2011.01.027

16. Brucato A, Imazio M, Gattorno $\mathrm{M}$, et al. Effect of anakinra on recurrent pericarditis among patients with colchicines resistance and corticosteroid dependence: the AIRTRIP randomized clinical trial. JAMA 2016; 316: 1906-12; https://doi.org/10.1001/jama.2016.15826

17. Imazio M, Brucato $A, M a e s t r o n i ~ S$, et al. Risk of constrictive pericarditis after acute pericarditis. Circulation 2011; 124: 1270-5; https://doi.org/10.1161/CIRCULATIONAHA.111.018580

18. Imazio M, Brucato A, Adler Y, et al. Prognosis of idiopathic recurrent pericarditis as determined from previously published reports. Am J Cardiol 2007; 100: 1026-8; https://doi.org/10.1016/j. amjcard.2007.04.047 